\title{
"Fluidic Diode" for Passive Unidirectional Liquid Transport Bioinspired by the Spermathecae of Fleas
}

\author{
Gerda Buchberger ${ }^{*}$, Alexander Kogler ${ }^{2}$, Agnes Weth ${ }^{1}$, Richard Baumgartner $^{2}$, Philipp Comanns ${ }^{3}$, \\ Siegfried Bauer ${ }^{2}$, Werner Baumgartner ${ }^{1}$ \\ 1. Institute of Biomedical Mechatronics, Johannes Kepler University Linz, Altenberger Str. 69, A-4040 Linz, Austria \\ 2. Institute of Experimental Physics, Department of Soft Matter Physics, Johannes Kepler University Linz, \\ Altenberger Str. 69, A-4040 Linz, Austria \\ 3. Institute of Biology II, RWTH Aachen University, Worringerweg 3, D-52074 Aachen, Germany
}

\begin{abstract}
We present a device for passive unidirectional liquid transport. The capillary channels used are bioinspired by the shape of the spermathecae (receptaculum seminis) of rabbit fleas (Spilopsyllus cuniculi) and rat fleas (Xenopsylla cheopis). The spermatheca is an organ of female fleas that stores sperm until suitable conditions to lay eggs are found. We translated and multiplied the natural form and function of a spermatheca to create a continuous capillary system from which we designed our microfluidic device based directly on the model from nature. Applying the Young-Laplace equation, we derived a theoretical description of local liquid transport, which enables model-guided design. We arranged the bioinspired capillaries in parallel and engraved them in poly(methyl methacrylate) (PMMA) plates by $\mathrm{CO}_{2}$ laser ablation. The fabricated structures transport soapy water passively (i.e., without external energy input) in the forward direction at velocities of about $1 \mathrm{~mm} \cdot \mathrm{s}^{-1}$ while halting the liquid fronts completely in the backward direction. The bioinspired capillary channels are capable of unidirectional liquid transport against gravity. Distance and velocity measurements prove the feasibility of the concept. Unidirectional passive liquid transport might be advantageous in technical surfaces for liquid management, for instance, in biomedical microfluidics, lab-on-chip, lubrication, electronics cooling and in micro-analysis devices.
\end{abstract}

Keywords: liquid diode, wetting, passive unidirectional liquid transport, capillary, bioinspired

Copyright $($ C The Author(s) 2018, corrected publication 03/2018. This article is published with open access at Springerlink.com.

\section{Introduction}

Existing approaches to unidirectional liquid transport on technical surfaces usually require an external energy source of vibration, electrical energy, radiation or similar ${ }^{[1-3]}$. Our "liquid diodes", in contrast, allow liquids to be transported passively (i.e., without external energy input) in a preferential direction while halting the liquid front in the reverse direction. The design is inspired by the spermathecae (receptaculum seminis) of specific kinds of fleas, such as the oriental rat flea (Xenopsylla cheopis) and the rabbit flea (Spilopsyllus cuniculi).

The spermatheca is an organ of the female flea where sperm of male fleas is stored after copulation until suitable conditions to lay eggs are found, i.e. after feeding on blood of hosts. Then the sperm is released from the spermatheca. The spermatheca is a flask- shaped organ with a globular base (bulga) that is connected via a diaphragm to a longer, bended narrow neck $(\text { hilla })^{[4,5]}$ (Fig. 1). In this organ, sperm can only enter and be released through the orifice (a sideways junction with sharp change in diameter) of the spermathecal duct (ductus spermathecae ${ }^{[6]}$ or ductus receptaculi seminis ${ }^{[7]}$ ). All parts have diameters in the range of $35 \mu \mathrm{m}$ to $90 \mu \mathrm{m}$ (Fig. 1).

Rothschild $^{[4]}$ analyzed the morphology of impregnated spermathecae of different species of fleas and described furthermore the arrangement of sperm in spermathecae. The spermatheca is gradually filled from the bulga to the hilla during mating $(\mathrm{s})^{[4]}$. In this process some sperm arrange themselves between the strigillae which are chitinous ridges located at part of the spermatheca's wall ${ }^{[4]}$. It seems fairly certain that the sperm is nourished when arranged in this way ${ }^{[4]}$.

While it is relatively clear that the spermatheca is

*Corresponding author: Gerda Buchberger

E-mail: gerda.buchberger@jku.at 

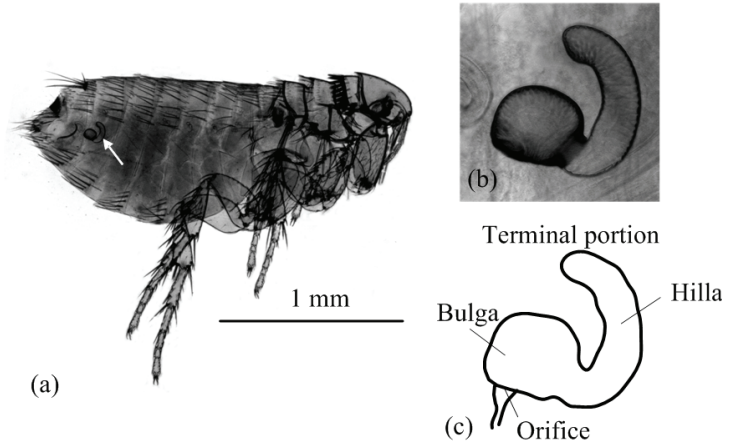

Fig. 1 Morphology of the spermatheca (receptaculum seminis) of the rat flea (Xenopsylla cheopis). (a) Overview of a female specimen. The receptaculum seminis is indicated by an arrow; (b) higher magnification of the receptaculum. The side length of this image is $200 \mu \mathrm{m}$; (c) schematic drawing of the receptaculum shown in $b$. The mouth of the orifice can hardly be seen in (b). The orifice discharges under pressure into the widened bulga, which terminates in the relatively narrow hilla ${ }^{[5]}$.

filled by pressure due to the ejaculation of the male flea ${ }^{[4,7-9]}$, it is still unresolved how the liquid containing the sperm is released when fertilisation of the eggs is intended $^{[4]}$. The spermatheca is a cuticular structure ${ }^{[4]}$, i.e. the walls are made mainly of chitin. Therefore, it is hard to assume that muscular contraction can completely squeeze out the liquid. Unfortunately, no clear evidence is available in the literature, if contractile cells are located in the spermathecae walls. However, Mitzmain observed faintly discernible movements of the spermatheca during coition ${ }^{[9]}$ which might suggest a change in shape induced by muscles or by the injection pressure of the male. For rabbit fleas (Spilopsyllus cuniculi) it was speculated that the cuticle of the bulga and of the tip of the hilla is more rigid than the cuticle of the middle part of the hilla ${ }^{[4]}$. There is indication that contractile filaments connecting the bulga and the tip of the hilla ${ }^{[6]}$ can bend the structure ${ }^{[4]}$, which would lead to either a slight volume reduction of the lumen or a kink in the structure at the aperture where the hilla opens to the bulga. The latter would rather stop any flow, while bending movements could dislodge the sperm from the strigillae $^{[4]}$ and readily jumble the sperm ${ }^{[4]}$ for potential capillary transport out of the spermatheca.

Additionally, it is tempting to assume that the spermathecal duct entering into the bulga at the orifice can be closed by means of contractile cells. These effects would lead to trapping of the sperm in the spermathecae after filling by ejaculation. We speculate that the release of sperm from the spermatheca is, at least in part, a passive process facilitated by the morphology of the spermatheca itself ${ }^{[4]}$. We propose that this intrinsic property of the spermatheca is given by a capillary system (Fig. 1): the straight capillary (hilla) and the conic capillary (bulga), which allow for bidirectional flow of sperm, and the sideways capillary junction (orifice), which passively supports the release of sperm by capillary forces. Due to the abrupt change in diameter the orifice is thought to require pressure for sperm to be injected. Thus, the capillary pressure will release sperm from the spermatheca and will hinder sperm to enter the spermatheca, if there is no additional input of external energy, i.e. in case no male flea injects sperm.

It has to be emphasized that this passive release is only a hypothesis which has to be challenged experimentally in order to clarify to which extent this passive liquid movement plays a significant role in the biological system. However, the morphology of the system inspired us to build microfluidic devices to (a) clarify if a passive directional liquid movement can be achieved with such a structure and (b) to design technically relevant microfluidic channels and systems for unidirectional liquid transport with a wide range of applications in practical liquid handling.

In a biomimetic approach, we used microscopic images to analyze the organ's natural morphology (Fig. 1), abstracted its shape, described the underlying working principle for unidirectional liquid transport and transferred it to a technically interesting polymer widely used in microfluidics, namely poly(methyl methacrylate) (PMMA). This study only deals with the passive part of sperm transport, where no male flea is involved. We do not investigate or try to mimic the active process of sperm injection into the spermatheca.

In the literature, various concepts for passive liquid transport with $^{[10-16]}$ or without ${ }^{[17-20]}$ unidirectionality have been reported. Asymmetric "sawtooth" or "ratchet-like" features on the surface of capillaries give rise to directionally biased fluid transport which is either passive $^{[18]}$ or powered by external energy sources ${ }^{[3]}$. Tilted fins on the sidewalls result in one easy and one hard wicking direction in open micro-channels ${ }^{[20]}$. Chemical modification in the form of oxidization ${ }^{[14]}$ or partially coated tubes ${ }^{[17]}$ can also lead to directional fluid 
transport. For single droplets, an anisotropic nanofilm serves as a means for inducing directional transport vibrationally ${ }^{[14,21]}$. Chaudhury and Whitesides ${ }^{[15]}$ demonstrated a water droplet (one to two microliters) that ran uphill over a distance of one centimeter due to a gradient in surface free energy. Kühn et al. fabricated microfluidic channels from the polymer poly(dimethylsiloxane) (PDMS) which transport fluid droplets directionally against gravity ${ }^{[14]}$. He et al. presented leaf-inspired microfluidic chips from agarose hydrogel which are capable of pumpless, i.e. passive, fluid transport even against gravity ${ }^{[22]}$; however, they were not tested with regard to their flow directionality. Capture silk of the cribellate spider (Uloborus walckenaerius) has the ability to collect water directionally due to a surface energy gradient and a difference in Laplace pressure which both are a result of structural features ${ }^{[16]}$. Artificial spider silk was fabricated and mimicked the structure and water collection ability of natural spider silk. Bioinspired heat pipe wicks with a low-level hierarchical structure together with integral wicks for different pipe sections improved directional fluid transport towards the evaporator ${ }^{[23]}$.

Devices for unidirectional liquid transport are commonly referred to as "liquid diodes",[11], "fluid diodes",[24] or "microfluidic diodes",[25,26] in analogy to the electronic components. In Refs. [25] and [26], a moveable disc and a flap, respectively, supported microfluidic diode characteristics, thus enabling active fluid flow in the forward direction, while blocking it in the backward direction. Large-area coating of a porous cellulose substrate yields a passive fluid diode with flow direction normal to the surface ${ }^{[24]}$. Plamadeala et al. ${ }^{[27]}$ fabricated microstructures bioinspired by micro-ornamentation on flat bugs (Dysodius lunatus). These structures with a feature size of several tens of micrometers transport oil directionally in a closed capillary channel.

Microfluidic devices for unidirectional passive liquid transport bioinspired by the skin of a moisture-harvesting lizard, namely the Texas horned lizard (Phrynosoma cornutum), have been reported ${ }^{[10-13]}$. This concept involves the interconnection of at least two capillary channels ${ }^{[10,11]}$ which interact by oscillatory liquid fronts. A biomimetic capillary channel network based on this concept ${ }^{[12]}$ is thought to be advantageous when single capillary channel sites could be blocked by particles or due to fabrication inaccuracies.

\section{Materials and methods}

\subsection{Preparation and analysis of the biological sample}

We used a sample of a flea prepared according to a typical standard preparation procedure described in Ref. [28]. Since the sample was an item on loan (see "Acknowledgement") and therefore not prepared by ourselves, we do not give specific preparation steps here and refer the reader to the given literature. The images of the flea were taken by a camera (Moticam Pro 2050 from Motic) mounted on a microscope (Axiovert 25 from Zeiss).

\subsection{Aqueous test fluid of soapy water with red dye from Ponceau $S$}

In our tests, we used an aqueous solution of 0.72 vol $\%$ soap concentrate (DAWN ultra, Procter \& Gamble, USA) to which $3.85 \%$ filtrated aqueous red dye (0.5\% Ponceau $\mathrm{S}$ red, deionized water, 3 vol\% trichloroacetic acid) was added for higher contrast.

\subsection{Contact angle and surface tension measurements}

A commercially available setup (DataPhysics OCA-Series with the SCA20 Software for OCA) was used for both contact angle and surface tension measurement. We recorded the contact angle of the test fluid on plain PMMA surfaces (Evonik industries Plexiglas ${ }^{\circledR}$ extruded acrylic sheet conforming to ISO 7823-2) at an ambient temperature of $21^{\circ} \mathrm{C} \pm 1{ }^{\circ} \mathrm{C}$ and a relative humidity of $50 \% \mathrm{RH} \pm 5 \% \mathrm{RH}$, applying droplets of $2 \mu \mathrm{L}$ at a dosing rate of $1 \mu \mathrm{L} \cdot \mathrm{s}^{-1}$. We employed the static sessile droplet method in combination with elliptic fits. We started measurement directly after placing the droplet onto the polymer surface using a $500 \mu \mathrm{L}$ Hamilton syringe with a blunt end. Further, we used the pendant droplet method for surface tension measurement between liquid and ambient air at an ambient temperature of $21^{\circ} \mathrm{C} \pm 1{ }^{\circ} \mathrm{C}$ and a relative humidity of $50 \% \mathrm{RH} \pm 5 \%$ RH. The droplet volumes applied varied between $2.5 \mu \mathrm{L}$ and $3.1 \mu \mathrm{L}$. The soapy water test liquid (section 2.2) was measured to have a static contact angle $\theta$ of $56^{\circ}$ $\pm 2^{\circ}( \pm 4 \%, n=6)$ on PMMA and a surface tension $\gamma$ in the range of $24 \mathrm{mN} \cdot \mathrm{m}^{-1}$ to $30 \mathrm{mN} \cdot \mathrm{m}^{-1}$ at standard laboratory conditions. In order to reduce the contact angle of 
the test liquid further, we changed the surface energy of the PMMA by sputter-coating a thin layer of gold. To this end, we used a BAL-TEC SCD 005 Cool Sputter Coater (BAL-TEC AG, EM-Technology and Application, Liechtenstein). We coated the samples for $40 \mathrm{sec}-$ onds applying a current of $25 \mathrm{~mA}$. This procedure resulted in a contact angle of $24^{\circ} \pm 2^{\circ}( \pm 9 \%, n=10)$.

\subsection{Biomimetic abstraction process of natural role model}

The morphology of the rat flea spermatheca suggests a passive transport mechanism for storage and release of sperm (Figs. 1 and 2). According to our hypothesis the shape of the spermatheca supports the release of sperm and requires pressure for injection of sperm (Fig. 2a). The hilla resembles a straight (A) and the bulga a conic capillary (B), respectively. Both are assumed to allow for bidirectional transport of sperm. Following a biomimetic approach their shapes are abstracted correspondingly, taking into account their functionalities; both are assumed to allow bidirectional transport. According to our hypothesis the conic bulga with its sideways orifice (C) facilitates the release of sperm and requires pressure for injection of sperm. It forms a singular transition point (C) with an abrupt widening of the capillary which hinders the sperm to flow into the spermatheca. In the device design for unidirectional liquid transport this transition point is responsible for halting of the liquid front.

In order to fabricate devices for passive, unidirectional liquid transport, we abstracted the shape of the spermatheca such that it forms the unit cell of the device. We then connected several of these unit cells to each other at the positions of the natural spermatheca's orifice and of the hilla tip (Fig. 2a), respectively, yielding a capillary channel of serial connected unit cells. Two connected unit cells in Fig. 2a illustrate the flow in the forward direction and halting of the liquid front in the backward direction. We thus use the principle of facilitated passive transport of sperm out of the spermatheca and hindered flow in the reverse direction to achieve unidirectional liquid transport in the forward direction of a microfluidic device.

Inspired by the natural model, we designed one unit cell of the structure (Fig. 2a) to consist of three func- tional unit cell components that are connected to each other (Fig. 2b): (A) a rotationally symmetric straight capillary of approximately constant radius like the hilla, (B) a conic rotationally symmetric capillary like the bulga and (C) a junction of two rotationally symmetric capillaries with a sharp narrowing of the capillaries' radii (i.e., a sharp change in dimensions over a small distance) like the orifice. As already mentioned above, the latter, the transition point (C), is responsible for halting of the liquid front.

The junction is not placed at the end of the conic capillary, but in the middle or its upper third. Capillary forces are thought to cause fluid transport, since typical diameters of the natural spermatheca are in the range of $35 \mu \mathrm{m}$ to $90 \mu \mathrm{m}$. As further step of abstraction the channel pattern is up-scaled and designed with rectangular cross sections and half open in order to meet demands of future technical applications where there is a need for free access over the transport path.

We scaled the structure up by a factor of about twelve, comparing the maximum diameter found in the natural structure to the maximum width in the artificial one. We designed the structure as shown in Fig. 2 with a unit cell length of $2.4 \mathrm{~mm}$ and an opening angle $\alpha$ of $26.6^{\circ}$ (video 1 provided as supplementary material). The width of the straight capillaries was chosen to be $0.3 \mathrm{~mm}$. In our technical implementation, we ensured that inertia forces and gravitational forces are negligible by calculating the Bond number, the Reynolds number and the Weber number. In this case the capillary forces and viscous forces balance each other similar to the example discussed in Ref. [29] describing the dynamics of capillary flow in a capillary tube. We calculated the capillary number $C a=\mu v / \gamma$ to be about $4 \times 10^{-5}$, which is well below one. It compares viscous forces to surface tension forces ${ }^{[30]}$. Here, $\mu$ is the dynamic viscosity of the aqueous test liquid, $\gamma$ its surface tension against ambient air and $v$ the transport velocity. Furthermore, we estimated the ratios of gravitational and inertia forces to the capillary forces, captured by the Bond number (Bo $=\Delta \rho g h w / \gamma)$ and the Reynolds number $\left(\operatorname{Re}=\rho v d_{\mathrm{h}} / \mu\right)^{[30]}$. Furthermore, we calculated the Weber number $\left(W e=\rho v^{2} d_{h} / \gamma\right)$ which is a measure for the disruption of an interface under the action of inertial forces ${ }^{[31]}$. Here, $\Delta \rho$ denotes the difference between the density of liquid and 
(a) Spermatheca

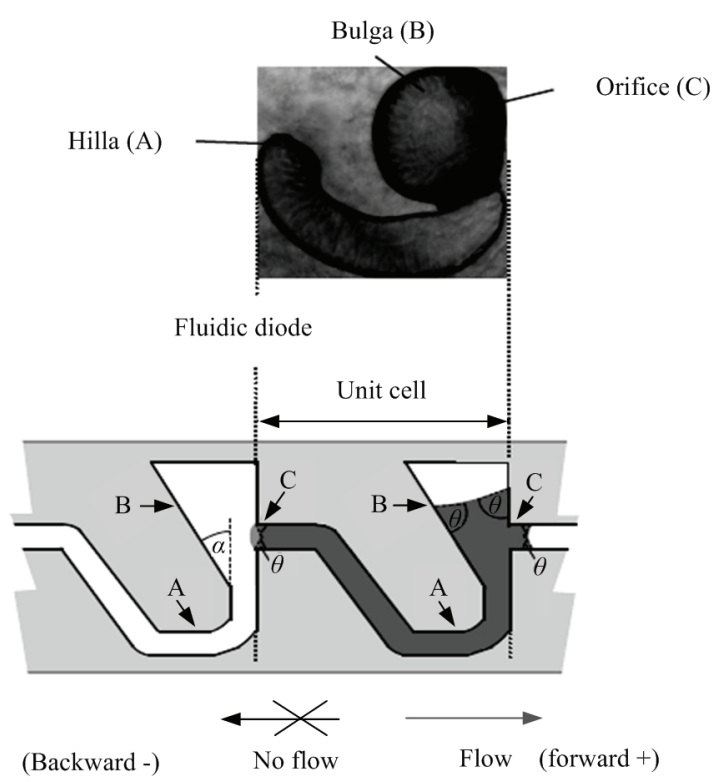

(b) Unit cell components

A

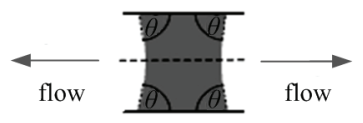

B
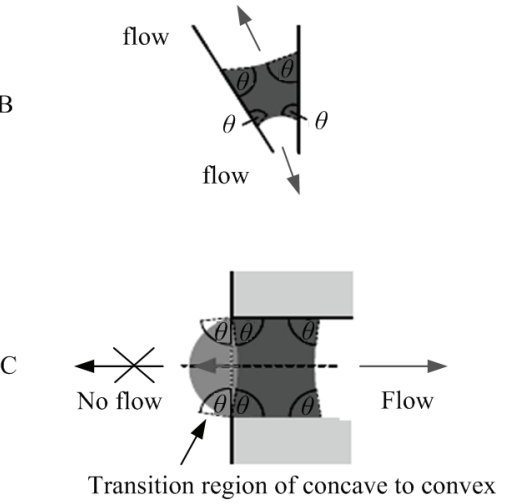

Fig. 2 Schematic illustration of a "fluidic diode" for passive unidirectional liquid transport bioinspired by the spermathecae of fleas. (a) The shape of the spermatheca is abstracted in order to form the unit cell of the fluidic diode for a liquid with a contact angle $\theta$. For device design, several of these unit cells are connected to each other; (b) elementary components of one unit cell: (A) a straight capillary channel for bidirectional flow, (B) a conic capillary channel with a small angle of slope $\alpha$ for bidirectional flow and (C) a sideways junction to the conic capillary with an abrupt widening forming a singular transition point, which results in unidirectional flow.

air, $g$ the gravity acceleration, $h$ the height of the liquid column in the conic capillary channel, $w$ the width of the conic capillary channel at the transition point, $\gamma$ the surface tension, $v$ the velocity of the transported liquid, $d_{h}=4 w h /(w+2 h)$ the hydraulic diameter of the open rectangular capillary channel with a width $w$ and a depth $h$, and $\rho$ the density of the liquid. Calculation of these characteristic numbers yields $B o \approx 0.2, R e \approx 0.7$ and $W e \approx 2 \times 10^{-5}$, respectively. As typical for microfluidics, liquid flow is laminar due to the low Reynolds number of liquid transport; furthermore, gravitational forces exerted by the liquid column in the conic capillary channel are small compared to capillary forces because the Bond number is considerably smaller than 1 . The very low value of the Weber number shows that inertial forces are negligible compared to surface tension forces ${ }^{[31]}$. Therefore, mainly capillary forces induce directional liquid transport in the designed biomimetic structures.

\subsection{Fabrication of biomimetic PMMA structures}

We fabricated bioinspired "fluidic diodes" from poly(methyl methacrylate) (PMMA) plates by laser ablation using a $\mathrm{CO}_{2}$ laser with a main wavelength in the infrared range of light. To this end, we used a Trotec Speedy 300 TM laser cutter/engraver. This engraver has a $95 \mathrm{~W} \mathrm{CO}_{2}$ laser source with a main wavelength of $10.6 \mu \mathrm{m}$ in the infrared range of light. The laser beam was focused with a spot size diameter of $100 \mu \mathrm{m}$ on the surface of the demonstrator plates using a Trotec lens with a focal length of $3.81 \mathrm{~cm}$ (1.5 inches). The material of the plates was $6 \mathrm{~mm}$ thick Evonik industries Plexiglas ${ }^{\circledR}$ extruded acrylic sheet conforming to ISO 7823-2. The PMMA was laser-structured at a pulse energy of $2.26 \mathrm{~mJ}$ achieved by selecting an average laser power of $95 \mathrm{~W}$ and a pulse repetition rate of $42 \mathrm{kHz}$. The active feed rate of the laser beam was $1.065 \mathrm{~m} \cdot \mathrm{s}^{-1}$ (42 inches $\cdot \mathrm{s}^{-1}$ ), resulting in a pulse density of $1000 \mathrm{ppi}$. The effective ablation depth per single laser cycle was about $1 \mathrm{~mm}$ for most parts of the biomimetic structures. For more details on the geometry the reader is referred to section 2.6.

In order to reduce the contact angle of the test liquid on some samples, we changed the surface energy of the PMMA by sputter-coating a thin layer of gold. To this end, we used a BAL-TEC SCD 005 Cool Sputter Coater 
(BAL-TEC AG, EM-Technology and Application, Liechtenstein). We coated the biomimetic structures for 40 seconds applying a current of $20 \mathrm{~mA}$. This procedure resulted in a contact angle of $34^{\circ} \pm 6^{\circ}( \pm 18 \%, n=6)$.

\subsection{Optical Coherence Tomography (OCT) for 3D surface topography}

The 3D topography data of the fabricated device (Fig. 3) was accessed by OCT. Therefore, we used a commercially available Telesto SD-OCT system from Thorlabs (Lübeck, Germany) with a center wavelength of $1325 \mathrm{~nm}$. In this system, the axial and the lateral resolution in air are less than $7.5 \mu \mathrm{m}$ and approximate $15 \mu \mathrm{m}$, respectively. For image processing, e.g. for image filtering (i.e. removal of dark and bright outliers) and calculation of topography data, we applied the freeware Fiji. The topography data shown in Fig. 3a was smoothed by a median filter in Matlab (medfilt2-function with a square field of $0.07 \mathrm{~mm} \times$ $0.07 \mathrm{~mm}$ ). We calculated the depth profile of the
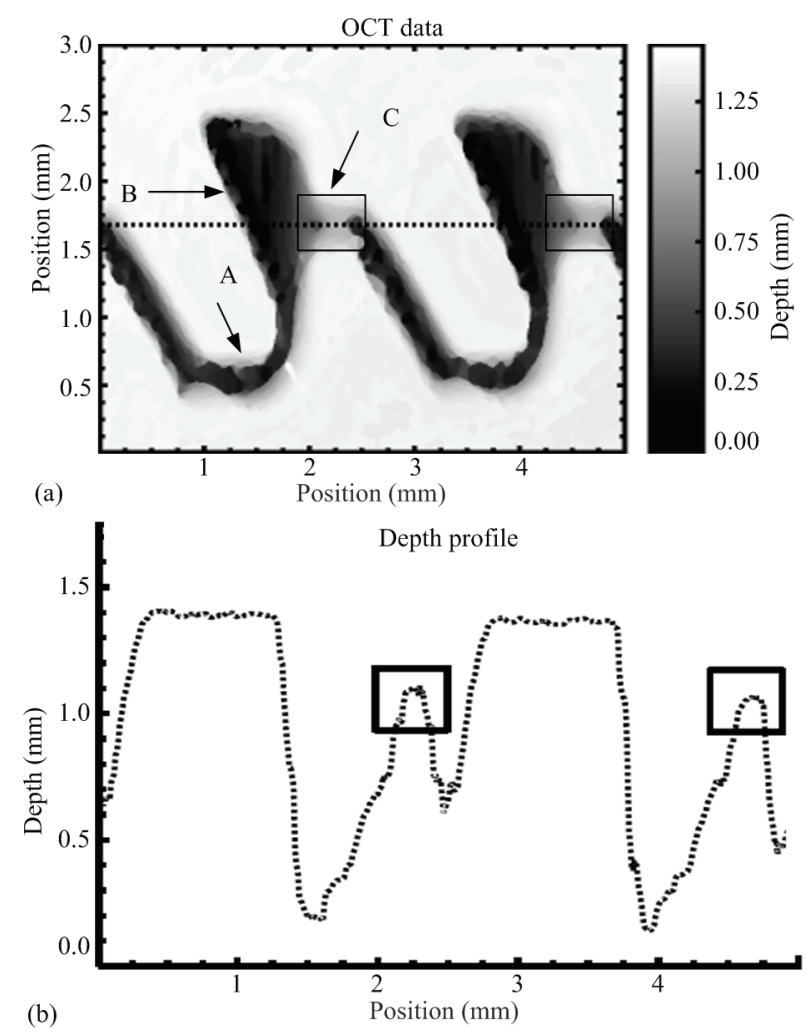

Fig. 3 Characterization of fabricated biomimetic "fluidic diode". (a) 3D surface topography accessed by OCT; (b) depth profile of the structure along the black dotted line in (a). The capillary channel is shallower in the region near the transition point marked by black squares. fabricated structure (Fig. 3b) along the black dotted line in Fig. 3a averaging the data $\pm 0.125 \mathrm{~mm}$ above and below the line. The chosen laser parameters and layout led to capillary channels that are shallower near the transition point. The resulting structure has a typical depth of about $1 \mathrm{~mm}$ except for the region around the transition point with a depth of about $0.3 \mathrm{~mm}$ (Figs. 3a and $3 b)$. This is important in order to pin the liquid front at the transition point effectively without the site being overcome by unwanted flow at the floor and walls of the capillary channel.

\subsection{Optical analysis of unidirectional fluid transport}

Videos of the liquid fronts served as an optical method for distance and velocity measurements. We conducted the measurements using a Full-HD HighSpeed Camcorder (GC-PX10, JVC KENWOOD Corporation) with a resolution of 1920 pixels $\times 1080$ pixels and a frame rate of $50 \mathrm{fps}$. For video editing we employed Windows Movie Maker 2012. In our video analysis, we first stripped down the video to single images using the free software Avidemux 2.6.10 and then analyzed the images using the freeware Fiji. The measurement setup was custom-designed to measure unidirectional liquid transport against gravity on the bioinspired structures. The setup included a table for specimens hinged to a frame with a goniometer in order to adjust the angle of inclination. A plumb line allowed reading out the angle of inclination.

\subsection{Linear fits and standard deviations of parame- ters}

A device with only a single bioinspired capillary channel was employed to record the distance- versus-time curves (video 2 of the supplementary material is an example measurement video). The single capillary channel was used to prevent excessive capillary channel overflow and possible crosstalk between several capillary channels. Every second frame of the videos was evaluated, which resulted in a frame rate of $25 \mathrm{fps}$. We analyzed the flow in the forward direction for as long as the structure completely halted the liquid flow in the backward direction. After some time, the test liquid spread onto the unstructured polymer surface next to the capillary channel and then flowed back into the capillary 
channel, which caused backward flow. We applied robust linear regression to the distance relative to time for each of the five measurement cycles. To this end, we used the "robustfit"-function in Matlab with the default weighting function "bisquare". With the help of this function, we obtained the linear slopes of the distances relative to time and their standard deviations. Since the relation between distance and time was found to be linear, the slopes gave averaged velocity values for each measurement cycle. From these values, we calculated the mean velocity and its standard deviation for all five cycles by applying the Gaussian law of propagation of uncertainty for independent variables.

\subsection{Algorithm for video analysis of unidirectional passive fluid transport}

For video analysis, we first cropped the video to the region of interest by the freeware "Free Crop Video", then we stripped down the videos to single images and after that we processed the image sequences by the commercially available software Matlab 2015a. For image analysis, we took only one RGB layer of each image, namely the layer with the highest contrast of the test fluid to the background. Then we performed a background subtraction by using an averaged image of the first images in the sequence, i.e. the images before liquid had been dispensed onto the demonstrators. We blurred the resulting images by a Gaussian smoothing function in order to get rid of unwanted noise. We applied a threshold value to the resulting image, which set the pixel values of the wetted area to " 1 " and the other pixel values to " 0 ". By generating this binary image, we succeeded in segmenting the wetted area on the bioinspired structures from the non-wetted area. Using these results, we calculated the boundaries of the wetted area versus time and liquid front movement versus time, both in forward direction.

\section{Theory and calculation}

\subsection{Modeling: Young-Laplace equation and its im- plications for conic capillaries}

If a liquid slug is transported in a capillary of arbitrary shape, it forms a meniscus at each free liquid-air interface. The shapes of the menisci depend on the contact angle $\theta$ between solid, liquid and ambient air and on the geometry, that is, on the radius $r$ in the case of a rotationally symmetric capillary and on the angle of slope $\alpha$ of the capillary's wall.

The Young-Laplace equation describes the capillary driving pressure difference

$$
\Delta p=\gamma\left(\frac{1}{r_{1}}+\frac{1}{r_{2}}\right),
$$

where $r_{1}$ and $r_{2}$ denote the principal radii of curvature of the liquid meniscus. A positive capillary pressure difference $\Delta p$ will lead to passive liquid transport.

In nature, all parts of the spermatheca are rotationally symmetric in a good approximation. However, for future technical applications - especially in the field of microfluidics and lab-on-a-chip - free access over the entire transport distance is desirable (Fig. 4). To this end, we use open capillary channels (Fig. 4a) rather than rotationally symmetric capillaries.

Eq. (1) remains the same for both types of structure, which is why similar functional principles apply. The capillary channels have a spatially varying width $w(x)$ (Fig. 4b) and a finite depth $h(x)$ (Fig. 4c). In this case, the upper interface between air and liquid distorts the liquid front; therefore, the aspect ratio $a(x)=h(x) / w(x)$ influences the capillary driving pressure. The angle of slope $\alpha$ is positive, negative and zero for widening, narrowing and straight capillary channel sections, respectively. For conic capillary channels, as used in our case, the symmetry plane is inclined by $\alpha / 2$ with respect to the horizontal plane. While the meniscus between the sidewalls can be either concave or convex, depending on $\alpha$ and $\theta$ (Fig. 4b), the meniscus at the ambient air interface is

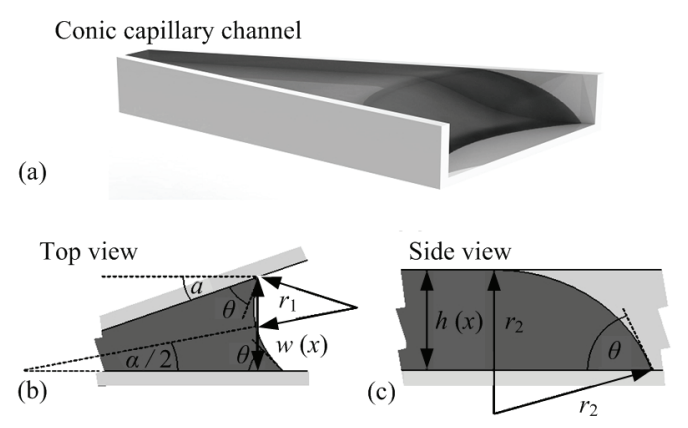

Fig. 4 Liquid slug with a contact angle $\theta$ in a conic capillary channel of finite depth $h(x)$, with a spatially dependent width $w(x)$ and with an angle of slope $\alpha$. (a) Three-dimensional view; (b) concave liquid front with a radius of curvature $r_{1}$ between the sidewalls; (c) convex liquid front with a radius of curvature $r_{2}$ at the interface to ambient air. 
always convex (Fig. 4c) ${ }^{[12]}$. Inserting the corresponding radii of curvature $r_{1}=w(x) /(2 \cos (\theta+\alpha / 2) \cos (\alpha / 2))$ and $r_{2}=-h(x) /(1-\cos \theta)$ into the Young-Laplace equation (Eq. (1)) yields a pressure difference of

$$
\begin{aligned}
& \Delta p(x) \\
& =\gamma / h(x) \cdot(-1+\cos \theta+2 \alpha(x) \cos (\alpha / 2) \cos (\theta+\alpha / 2)) .
\end{aligned}
$$

\subsection{Modeling: theoretical description of unit cell components based on the results for conic cap- illaries}

The driving pressure difference in the conic capillary channel (Eq. (2)) expresses all three functional unit cell components (A, B and C) of flea-inspired capillary channels, describing their role in unidirectional liquid transport.

\subsubsection{Straight capillary - unit cell component of type A}

Inserting $\alpha=0$ for straight capillaries into Eq. (2) results in equal positive pressure differences $\Delta p_{\text {straight }}{ }_{ \pm}=$ $\gamma / h_{\text {straight }}\left(-1+\cos \theta\left(1+2 \alpha_{\text {straight }}\right)\right)>0$ in both directions for concave menisci only, that is, for liquid-material combinations with $\cos \theta>1 /\left(1+2 \alpha_{\text {straight }}\right)$.

\subsubsection{Conic capillary - unit cell component of type B}

According to Eq. (2), type B capillaries give rise to unequal driving pressure differences $\Delta p_{\text {conic }, \pm}=$ $\gamma / h_{\text {conic }} \cdot\left(-1+\cos \theta+2 \alpha_{\text {conic }}(x) \cos (\alpha / 2) \cos (\theta \pm \alpha / 2)\right)$ in the forward and the backward direction. For a given liquid-solid-gas combination with a contact angle of $\theta$, the angle of slope $\alpha$ must be chosen such that liquid transport in the forward direction is sustained. The pressure difference in the forward direction must therefore be positive $\Delta p_{\text {conic, }+}>0$, which means that the meniscus has to be concave. This requires both menisci to be concave, resulting in positive driving pressures $0<\Delta p_{\text {conic, },+}<$ $\Delta p_{\text {conic,- }}$ in both directions.

\subsubsection{Junction with sharp change in capillary width - unit cell component of type $\mathrm{C}$}

For junctions, the dimensions change sharply or even abruptly, which leads to high driving pressure and rapid transport in the forward direction. According to Eq. (2), the upper limit for the positive pressure differ- ence is $\Delta p_{\text {junction, }}=2 \gamma / w_{\text {straight }}>0$. The singular transition point at the junction causes a convex liquid front to form in the backward direction and thus a negative pressure difference with a lower limit of $\Delta p_{\text {junction,- }}$ $=-2 \gamma \sin \theta / w_{\text {straight }}<0$. Therefore, the transition site stops the liquid front in the backward direction. This effect is similar to the one used in microfluidic stop valves ${ }^{[32]}$. Junctions of type $\mathrm{C}$ are therefore a necessary constraint to guarantee unidirectional liquid transport. The junctions are recommended to be located above the middle or within the upper third of the conic capillary channels. This ensures sufficient distance between the liquid meniscus and the channel walls to prevent the liquid from overcoming these junctions (i.e. halting points) in the reverse direction by wetting the neighboring walls of the conic capillary channel. It is important, to design these transition points safely according to the above recommendation so that the liquid halts in backward direction even in the case of external perturbations, fabrication inaccuracies, material roughness, edge effects ${ }^{[33,34]}$ and subjection to back-pressure. Furthermore, this design guideline makes the reservoir formed by the conic capillary channel large enough to serve as a liquid source for the next unit cell; this source keeps the time span for the filling of one unit cell approximately constant. The conic capillary channel of the fabricated structure is designed to have a total volume of $\approx 0.9 \mathrm{~mm}^{3}$ or $\approx 0.9 \mu \mathrm{L}$; the liquid inside can fill the whole straight capillary channel A; if the conic capillary channel would be filled up only partly till the transition point $\mathrm{C}$ of the next unit cell, the liquid volume inside was $\approx 0.3 \mathrm{~mm}^{3}$ or $\approx 0.3 \mu \mathrm{L}$. At this bifurcation point the liquid starts to flow into the shallower part of the straight capillary channel A (Fig. 3) near the transition point.

\subsubsection{Design guideline for open capillaries and remarks} on liquid transport against gravity

If one meniscus is concave while the other is convex, the two contributions to the capillary driving pressure in Eq. (2) will differ in sign. However, liquid transport requires positive capillary driving pressure. From Eq. (2) we derive a threshold aspect ratio

$$
\alpha(x)>(1-\cos \theta) /(2 \cos (\alpha / 2) \cos (\theta+\alpha / 2))>0,
$$

for a given contact angle $\theta$ and a given angle of slope $\alpha$, 
below which the structure will not transport liquid as a droplet will form that does not enter the capillaries. Importantly, the surface tension $\gamma$ between liquid and ambient air does not influence the sign of the driving pressure but only its absolute value. The upper limit for contact angles must be taken into account in the technical implementation. The threshold aspect ratio for the fabricated structure was calculated to be $a_{s}(x) \approx 0.6$ for the used test liquid. The aspect ratio of the straight capillary channel (of type A) was designed to be approximately 3.3, resulting from a width of approximately $300 \mu \mathrm{m}$ and a depth of approximately $1 \mathrm{~mm}$. The conic capillary channel (of type B) cannot be described by a single aspect ratio, but was designed to have aspect ratios ranging from a minimal value of approximately 1 at its widest part to approximately 3.3 at its narrowest part, where it is connected to the straight capillary channel. Near the transition point (of type C) the straight capillary channel is shallower with a depth of approximately $350 \mu \mathrm{m}$, yielding an aspect ratio of approximately 1.2.

Although the sign of the capillary driving pressure is not influenced by the surface tension $\gamma$ according to Eq. (2), its magnitude is proportional to it, which means that the capillary driving pressure difference (i.e., the flow dynamics and therefore the flow velocity) increases with the surface tension of the liquid. Similarly, the contact angle value will change the magnitude of the driving pressure difference, which again is described by Eq. (2): The smaller the contact angle is, the smaller the negative contribution of the convex curvature of the meniscus in height-direction $\Delta p_{n}(x)=\gamma / h(x) \cdot(-1+\cos \theta)$ is; at the same time, the positive contribution of the concave curvature of the meniscus $\Delta p_{p}(x)=\gamma h(x) \cdot 2 \mathrm{a}(x) \cos (\alpha / 2) \cos (\theta+\alpha / 2) \quad$ gets larger. Therefore, a reduction of the contact angle results in an increase of flow dynamics with a higher transport velocity in forward direction. However, too low contact angles will decrease the negative capillary driving pressure difference in backward direction so much that the liquid front will overcome the transition points (see section 3.2.3) due to perturbations, fabrication inaccuracies and boundary effects.

A positive capillary driving pressure can compensate for a certain negative hydrostatic pressure exerted by the gravitational force onto the mass of the liquid slug in the capillary. This means that unidirectional liquid flow even works against gravity for a certain height of capillary rise, which was verified by the experiments presented in the next section.

\section{Results and discussion}

\subsection{Results}

To test the manufactured structure, we applied an aqueous solution of $0.72 \mathrm{vol} \%$ soap concentrate to which aqueous red dye from Ponceau S (3.85 vol\%) was added for better visibility. We dispensed a droplet of $200 \mu \mathrm{L}$ onto the structure (Fig. 5). Our video analysis revealed that all eight capillary channels on the device transported the test fluid in the forward direction at a velocity in the range of $1 \mathrm{~mm} \cdot \mathrm{s}^{-1}$ while stopping the liquid fronts in the backward direction for test distances of about $26 \mathrm{~mm}$ in both directions. As predicted theoretically, the liquid front was pinned at halting sites in the backward direction. This proves the unidirectional liquid transport capability of the device.

We used a demonstrator with a single bioinspired capillary channel for accurate testing of distances covered relative to time (Fig. 6, video 2 provided as supplementary material). This approach prevents excessive capillary channel overflow and possible crosstalk between several capillary channels. We dispensed a droplet of $50 \mu \mathrm{L}$ of the test liquid onto the single capillary channel and recorded five consecutive measurement cycles by means of a video camera. We evaluated the data until backward flow occurred. To this end, the traveling time needed for each unit cell was measured. On the global scale, the data indicates a linear relation between distances traveled by the fluid fronts in the forward direction and traveling time (Fig. 6a). Therefore fluid transport over large distances is faster than in standard straight capillaries, where the distance traveled by the meniscus is related to the square root of time ${ }^{[29]}$. This is due to the reservoir formed by the conic capillary

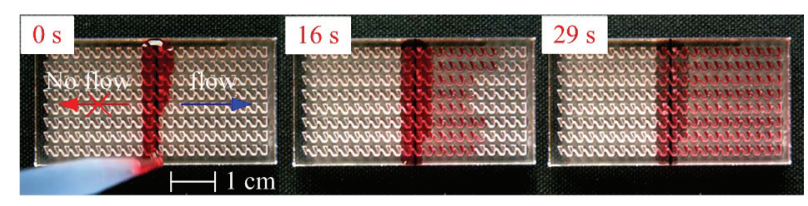

Fig. 5 Unidirectional liquid transport in the forward direction on a bioinspired structure on an even surface. For testing we dispensed $200 \mu \mathrm{L}$ of a test liquid of dyed soapy water onto the structure. 

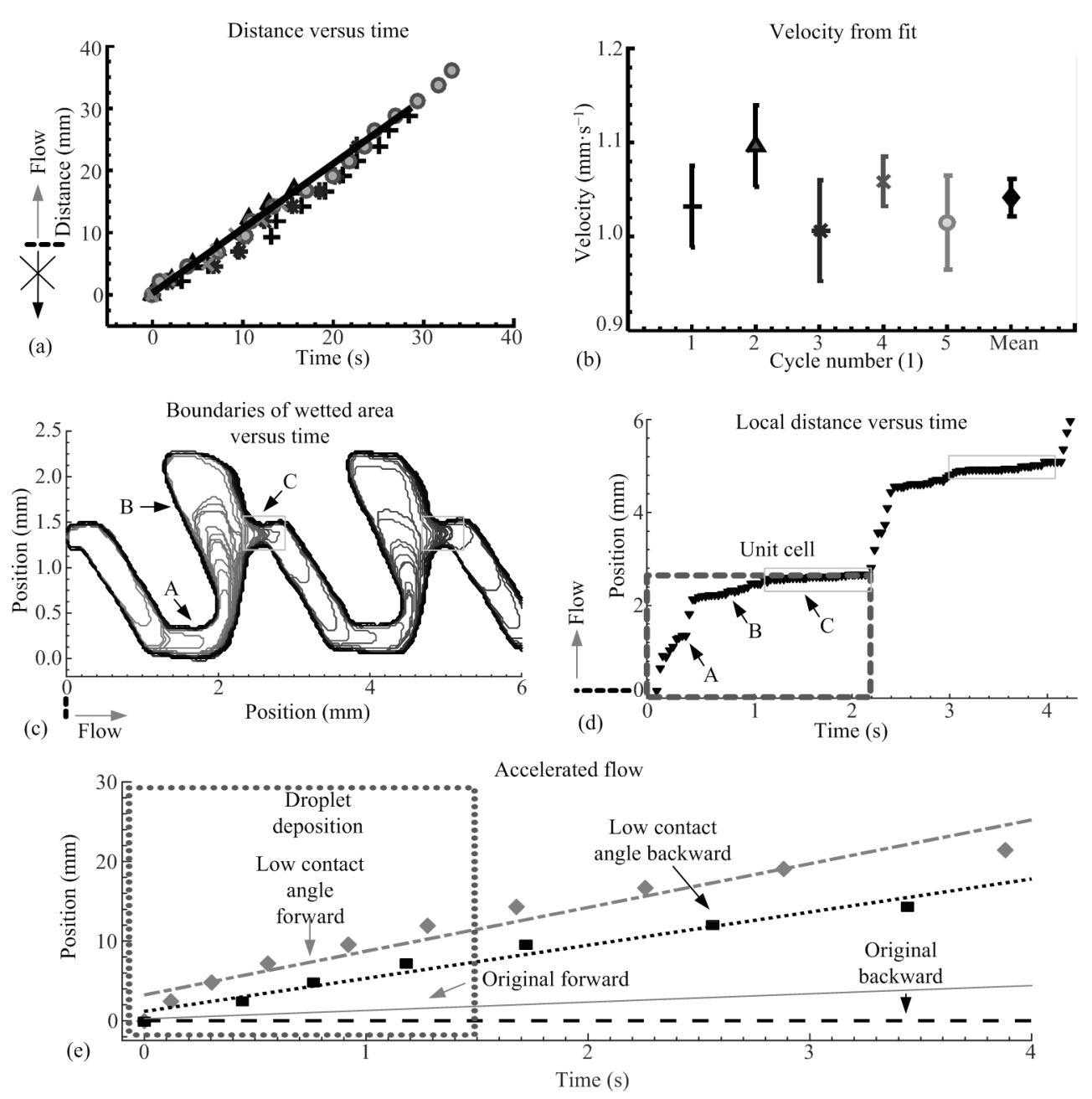

Fig. 6 Liquid transport measurement data for bioinspired structure. (a) Measurement data of distance against time with the mean linear fit (black, solid line) applying droplets of $50 \mu \mathrm{L}$ of the soapy water test liquid; (b) velocity values determined from the linear fits of the five single transport cycles in (a) and mean velocity value calculated from all fits; (c) boundaries of the wetted area (gray lines) in two unit cells; (d) local distance versus time. The measurement points correspond to the boundaries in (c); (e) increase of the velocity by reducing the contact angle by about $20^{\circ}$ resulted in directional instead of unidirectional liquid transport.

(B) (Fig. 2) which is deeper than the connective straight capillary channel (Fig. 3). This reservoir acts as a liquid source for the following unit cell; the straight capillary (A) originates directly at the sideways junction (C) to this reservoir: At the initial point of each unit cell, i.e. at the starting point of the straight capillary, liquid is extracted directly from the associated reservoir formed by the conic capillary of the previous unit cell. Therefore, the liquid front only drags the liquid slug over one unit cell. To be exact, the liquid slug at the beginning of the unit cell is fed by the liquid reservoir of the previous unit cell and is then dragged through the straight capillary channel (type A) to the reservoir formed by the conic capillary channel (type B); then it fills the conic reservoir. Thus, the liquid front will not slow down, if it travels over several unit cell lengths. The traveling time through each unit cell of the structure stays approximately constant, which leads to the measured linear relation between distance and time. However, on the local scale the transport velocity shows a more complex dependency on time which was expected (Figs. 6c and 6d). This complex behavior was studied by recording the boundaries of the wetted area versus time in time steps of $1 / 30 \mathrm{~s}$. Fig. 6c shows exemplary data for droplets of $50 \mu \mathrm{L}$ of the soapy water test liquid in two unit cells. Fig. 6d illustrates the complex time dependency of liquid movement: The liquid moved fastest in the straight capillary channel in parallel to the forward direction. It slowed down in the conic capillary channels, since most transport occurred normal to the forward direction and 
furthermore liquid transport was slower due to the widening of the capillary channel (see section 3.2.2.). Near the shallow transition point liquid movement slowed down even further. However, on the global scale the transport velocity was in the range of $1 \mathrm{~mm} \cdot \mathrm{s}^{-1}$, since the time the liquid front needs for one unit cell stays approximately constant.

By means of linear regression we determined the corresponding fit curves and velocity values for each measurement cycle (Fig. 6b). From all linear fits, a mean fit curve and a mean velocity value of $1.04 \mathrm{~mm} \cdot \mathrm{s}^{-1} \pm$ $0.02 \mathrm{~mm} \cdot \mathrm{s}^{-1}( \pm 2 \%)$ were calculated. Based on the theoretical results (see section 3 ), we find that the flow can be accelerated by lowering the contact angle and by increasing the surface tension of the test liquid. Increase of the velocity by reducing the contact angle by about $20^{\circ}$ on the gold-coated device led to the loss of the unidirectionality (Fig. 6e): fluid transport becomes directional instead with average velocities of $5.5 \mathrm{~mm} \cdot \mathrm{s}^{-1}$ in forward direction and $4.5 \mathrm{~mm} \cdot \mathrm{s}^{-1}$ in backward direction; these values were calculated by linear regression. However, distance relative to time does not seem to be linear anymore which means that transport velocity is not constant. A reason for this is that the liquid already spreads several millimeters during droplet deposition which means that the liquid volume serving as source is smaller at the beginning of the observation time period. Above a certain threshold contact angle, the biomimetic structure completely stops transporting the liquid, which is in accordance with the theoretical description presented. However, these effects require thorough systematic analysis, which is the subject of ongoing investigations. In general, we observed typical transport velocities of a few $\mathrm{mm} \cdot \mathrm{s}^{-1}$ in the case of unidirectional and directional liquid transport on the tested devices.

The fabricated structures allow directional liquid transport also against gravity (Fig. 7). Insets show the orientation of the unit cell in the test configurations. We used $50 \mu \mathrm{L}$ of test liquid to demonstrate horizontal (i.e., normal to the gravitational force) unidirectional transport (Figs. 7a and 7c). The conic capillary channel points against the gravity vector in Fig. 7a and in the direction of the gravity vector in Fig. 7c, respectively; correspondingly, the feeder loop made of straight capillary channels is aligned downwards in Fig. 7a and upwards in
Fig. 7c. At the local scale of the conic capillary channel, fluid will (1) either flow against gravity into the widening conic capillary channel (Fig. 7a) or (2) in parallel to the gravity vector into the widening conic capillary channel (Fig. 7c); due to the larger width and therefore smaller aspect ratios, the capillary driving pressure difference is smaller in the conic capillary channels than in the straight ones (Eq. (2) in section 3.1). In the first case illustrated by Fig. 7a, hydrostatic pressure caused by gravity will impede filling of the conic channel by capillary forces, while in the second case illustrated by Fig. 7c this process will be facilitated by hydrostatic pressure. Both configurations are studied experimentally in order to show the device's capability of global fluid transport normal to the gravity vector in either case. However, the structures work slightly better with straight capillaries pointing downwards (Fig. 7a) than with straight capillaries pointing upwards (Fig. 7c). In the backward direction, the device halts a test liquid volume of $50 \mu \mathrm{L}$ even against gravity (Fig. 7b). The gravitational force accelerates liquid transport in the forward direction (Fig. 7d). We used a custom-designed measurement setup for the investigation of flow against gravity at a specific angle of inclination in addition to the demonstration shown in Fig. 7. A wide range of angles of inclination can be adjusted. Applying a droplet of $90 \mu \mathrm{L}$ of the test fluid, we found that the unidirectional

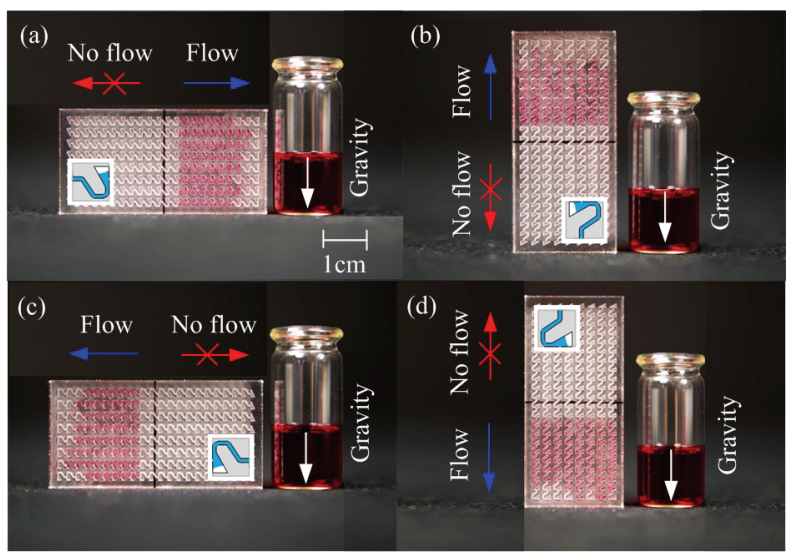

Fig. 7 Unidirectional transport of a $50 \mu \mathrm{L}$ dyed soapy water droplet against gravity on our biomimetic structure. Insets show the orientation of the unit cell with regard to the gravity vector. (a) Horizontal unidirectional transport (i.e., normal to the gravitational force); (b) liquid fronts are held vertically against gravity; (c) horizontal unidirectional transport with the capillary channels rotated by $180^{\circ}$ compared to (a); (d) vertical unidirectional transport with the forward direction parallel to the gravitational force. 
flow in the test structure can withstand an angle of inclination of $25^{\circ}$ for the test distance of $26 \mathrm{~mm}$ (video 3 provided as supplementary material). However, the gravitational force will slow down fluid transport.

\subsection{Discussion}

Our bioinspired "liquid diodes" for unidirectional transport are simple in design; there is no need for movable parts ${ }^{[25,26]}$, porous substrate ${ }^{[24]}$, closed capillary channels $\mathrm{s}^{[27]}$ or chemical modification of the surface ${ }^{[14,17,24]}$. In contrast to most concepts described in previous studies, we report unidirectional transport even against gravity. We can transport considerably larger fluid volumes than reported in Refs. [14], [15] and [27], where single droplets of a few $\mu \mathrm{L}$ were used. Our device completely halts the liquid front in the reverse direction like those presented in Refs. [11] and [12], which require oscillatory movement of the liquid front in two interconnected capillary channels or a capillary channel network. In our approach, however, we require no oscillatory fluid movement normal to the forward direction, since there is no need for interconnection. This allows denser arrangement of the capillary channels. We found a linear relation between distance traveled by the meniscus and traveling time, which means that the transport velocity of about $1 \mathrm{~mm} \cdot \mathrm{s}^{-1}$ is constant. This is due to the reservoir formed by the conic capillary. In contrast, transport velocities in devices bioinspired by the Texas horned lizard's skin $^{[10-13]}$ or velocities in traditional capillaries ${ }^{[29]}$ decrease significantly with time.

Further, our concept is preferable when an increased volumetric flow rate is desired due to the previously discussed denser arrangement and higher flow velocity. The presented design is also beneficial for fabrication, since the devices are more tolerant of variations in geometry and wetting properties of the fluid-material combination used. As our results show, the functionality of our devices is given by their shape only; however in nature there may be an additional active mechanism which results from contraction of tissue $^{[4]}$. Potential applications of the presented "fluidic diodes" arise wherever directional passive liquid transport is advantageous, for instance, in biomedical microfluidics, lab-on-a-chip and micro-analysis devices $^{[12,19,20,30,35,36]}$ as well as in electronics cooling ${ }^{[37]}$ and lubrication ${ }^{[11,13,38]}$.

It might be interesting in some applications to fabricate the biomimetic capillary channels on a smaller scale, e.g. ten times smaller. During down-scaling, it has to be ensured that the aspect ratio of the open capillary channels safely fulfills the condition of Eq. (3). It has to be noted that capillary channels with constant height do not possess reservoirs and will not show constant flow velocities as the devices presented in this work. Furthermore, they are more sensitive to the fluid parameters with regard to the halting site because in this case the capillary channel will not widen in the third dimension; this decreases the negative stopping pressure. For the fabrication of smaller structures, we recommend the techniques of two-photon polymerization ${ }^{[27,39,40]}$, soft lithography ${ }^{[25,41-43]}$, hot-embossing ${ }^{[44]}$, laser- ablation $^{[10,12]}$, replica-molding ${ }^{[45-47]}$ from masters, e.g. made by laser-ablation, or photolithography and dry etching ${ }^{[32]}$. Soft lithography is very popular in microfluidics ${ }^{[43]}$ : it includes a family of techniques involving a soft polymeric mold such as a poly(dimethyl siloxane) (PDMS) replica from an original hard master and has already been successfully used in biomimetic sensors ${ }^{[42]}$. Hot-embossing ${ }^{[44]}$ and replica-molding ${ }^{[46]}$ are known to be compatible with large scale roll-to-roll production. Biomimetic microfluidic diodes have been so far fabricated by laser-ablation in PMMA ${ }^{[12]}$ as well as in steel ${ }^{[10]}$. Two-photon polymerization is an emerging technology which is promising for three-dimensional structures with sizes of several tens of micrometers ${ }^{[39]}$ or smaller ${ }^{[40]}$. Therefore, it is in principle suited for small-scale microfluidics.

\section{Conclusion}

Bioinspired by spermathecae of fleas we described and fabricated "liquid diodes" for unidirectional liquid transport. The main functionality was found to result from junctions and conic capillary parts. Junctions of capillaries with an abrupt change in width or diameter serve as halting points and enable unidirectional transport. The conic parts contribute as local reservoirs through which the common properties of capillary flow could be overcome, i.e. the device exhibits an almost constant transport velocity on the global scale. Demonstrators from PMMA fabricated by laser-ablation 
showed transport velocities of the soapy water test liquid of about $1 \mathrm{~mm} \cdot \mathrm{s}^{-1}$ in the forward direction, while completely halting the liquid front in the backward direction. Using the Young-Laplace equation we described the underlying functionality in detail and gave basic design guidelines and restrictions for a wide range of material-liquid combinations. This includes, e.g., the contact angle as well as geometrical properties such as the aspect ratio of capillary channels. Our "liquid diodes" show unidirectional transport even against gravity. As the functionality is given by a passive mechanism, there is no need for external energy input. For specific applications, the volumetric flow rate can be increased by packing the capillaries densely. Since there are only few geometrical restrictions, the devices are tolerant of moderate variations in capillary dimensions and in wetting properties, which makes them suitable for large-scale production technologies. The design enables free access along the whole transportation path with potential for several fields of application, such as micro-analysis and lubrication.

\section{Acknowledgment}

We thank the Institute of Polymer Science at Johannes Kepler University Linz for providing the setup for surface tension and contact angle measurement, and the Biologiezentrum Linz for lending us the sample of the flea. We are grateful to Anna Stadler for her help with Fig. 1. Furthermore, we thank Thomas Fritz, Kurt Thaller B.Sc. and Dr. Andreas Buchsbaum from the company RECENDT GmbH for technical assistance. We acknowledge financial support from Kimberly-Clark Corporation and from the European Research Council within the Advanced Investigators Grant SoftMap (Soft Matter Physics Team). Financial support from the European Commission is acknowledged within the "LiNaBioFluid" project within the scope of H2020-FETOPEN-2014-2015-RIA. This research was further supported by the Austrian Research Promotion Agency (FFG) under contract number FFGP13830002/MicroNeedle.

Open Access This article is distributed under the terms of the Creative Commons Attribution 4.0 International License (http://creativecommons.org/licenses/by/4.0/), which permits use, duplication, adaptation, distribution and reproduction in any medium or format, as long as you give appropriate credit to the original author(s) and the source, provide a link to the Creative Commons license and indicate if changes were made.

\section{References}

[1] Woias P. Micropumps - Past, progress and future prospects. Sensors and Actuators, B: Chemical, 2005, 105, 28-38.

[2] Laser D J, Santiago J G. A review of micropumps. Journal of Micromechanics and Microengineering, 2004, 14, R35R64.

[3] Buguin A, Talini L, Silberzan P. Ratchet-like topological structures for the control of microdrops. Applied Physics A: Materials Science and Processing, 2002, 75, 207-212.

[4] Rothschild M L. Arrangement of sperm within the spermatheca of fleas with remarks on sperm displacement. Biological Journal of the Linnean Society, 1991, 43, 313-323.

[5] Traub R, Rothschild M L, Haddow J F. The Rothschild Collection of Fleas. The Ceratophyllidae: Key to the Genera and Host Relationships, Cambridge, University Press, UK, 1983.

[6] Mead-Briggs A R. The structure of the reproductive organs of the European rabbit-flea, Spilopsyllus Cuniculi (dale) (Siphonaptera). Proceedings of the Royal Entomological Society of London, Series A, General Entomology, 1962, 37, 79-88.

[7] Holland G P. Primary and secondary sexual characteristics of some Ceratophyllinae, with notes on the mechanism of copulation (Siphonaptera). Transactions of the Royal Entomological Society of London, 2009, 107, 233-248.

[8] Hsu M H, Wu W J. Off-host observations of mating and postmating behaviors in the cat flea (Siphonaptera: Pulicidae). Journal of Medical Entomology, 2001, 38, 352-360.

[9] Mitzmain M B. Some new facts on the bionomics of the California rodent fleas. Annals of the Entomological Society of America, 1910, 3, 61-82.

[10] Comanns P, Winands K, Arntz K, Klocke F, Baumgartner W. Laser-based biomimetic functionalization of surfaces: From moisture harvesting lizards to specific fluid transport systems. International Journal of Design and Nature and Ecodynamics, 2014, 9, 206-215.

[11] Comanns P, Buchberger G, Buchsbaum A, Baumgartner R, Kogler A, Bauer S, Baumgartner W. Directional, passive liquid transport: The texas horned lizard as a model for a biomimetic 'liquid diode'. Journal of the Royal Society Interface, 2015, 12, 20150415.

[12] Buchberger G, Hischen F, Comanns P, Baumgartner R, Kogler A, Buchsbaum A, Bauer S, Baumgartner W. Bio-inspired microfluidic devices for passive, directional liquid transport: Model-based adaption for different materials. Procedia Engineering, 2015, 120, 106-111. 
[13] Comanns P, Winands K, Pothen M, Bott R A, Wagner H, Baumgartner W. The Texas horned lizard as model for robust capillary structures for passive directional transport of cooling lubricants. Proceedings of SPIE - The International Society for Optical Engineering, 2016, 9797, 979711.

[14] Kühn P T, de Miranda B S, van Rijn P. Directed autonomic flow: Functional motility fluidics. Advanced Materials, 2015, 27, 7401-7406.

[15] Chaudhury M K, Whitesides G M. How to make water run uphill. Science, 1992, 256, 1539-1541.

[16] Zheng Y, Bai H, Huang Z, Tian X, Nie F Q, Zhao Y, Zhai J, Jiang L. Directional water collection on wetted spider silk. Nature, 2010, 463, 640-643.

[17] Weislogel M M. Steady spontaneous capillary flow in partially coated tubes. Aiche Journal, 1997, 43, 645-654.

[18] Extrand C W. Retention forces of a liquid slug in a rough capillary tube with symmetric or asymmetric features. Langmuir, 2007, 23, 1867-1871.

[19] Zimmermann M, Schmid H, Hunziker P, Delamarche E. Capillary pumps for autonomous capillary systems. Lab on a Chip, 2007, 7, 119-125.

[20] Feng J, Rothstein J P. One-way wicking in open micro-channels controlled by channel topography. Journal of Colloid and Interface Science, 2013, 404, 169-178.

[21] Malvadkar N A, Hancock M J, Sekeroglu K, Dressick W J, Demirel M C. An engineered anisotropic nanofilm with unidirectional wetting properties. Nature Materials, 2010, 9, 1023-1028.

[22] He J, Mao M, Li D, Liu Y, Jin Z. Characterization of leaf-inspired microfluidic chips for pumpless fluid transport. Journal of Bionic Engineering, 2014, 11, 109-114.

[23] Wang Q, Hong J, Yan Y. Biomimetic capillary inspired heat pipe wicks. Journal of Bionic Engineering, 2014, 11, 469-480.

[24] Mates J E, Schutzius T M, Qin J, Waldroup D E, Megaridis $\mathrm{C}$ M. The fluid diode: Tunable unidirectional flow through porous substrates. ACS Applied Materials and Interfaces, 2014, 6, 12837-12843.

[25] Adams M L, Johnston M L, Scherer A, Quake S R. Polydimethylsiloxane based microfluidic diode. Journal of Micromechanics and Microengineering, 2005, 15, 1517-1521.

[26] Sochol R D, Deeble C J, Shen V, Nakamura M, Hightower B J, Brubaker T A, Lee K Y, Gao S, Kim M, Wolf K T, Iwai K, Glick C C, Lee L P, Lin L. Single-layer microfluidic "disc" diodes via optofluidic lithography for ultra-low Reynolds number applications. The 17th International Conference on
Solid-State Sensors, Actuators and Microsystems (Transducers and Eurosensors XXVII), 2013, 2201-2204.

[27] Plamadeala C, Hischen F, Friesenecker R, Wollhofen R, Jacak J, Buchberger G, Heiss E, Klar T A, Baumgartner W, Heitz J. Bioinspired polymer microstructures for directional transport of oily liquids. Royal Society Open Science, 2017, 4, 160849.

[28] Herder V, Wohlsein P, Peters M, Hansmann F, Baumgartner W. Romeis - Mikroskopische Technik, Springer Berlin Heidelberg, Berlin, Heidelberg, Germany, 2015. (in German)

[29] Washburn E W. The dynamics of capillary flow. Physical Review, 1921, 17, 273-283.

[30] Berthier J, Silberzan P. Microfluidics in Biotechnology, 2nd ed., Artech House, Boston, USA, 2010.

[31] Berthier J, Brakke K A, Berthier E. Open Microfluidics, John Wiley \& Sons, Inc., Hoboken, NJ, USA, 2016.

[32] Zimmermann M, Hunziker P, Delamarche E. Valves for autonomous capillary systems. Microfluidics and Nanofluidics, 2008, 5, 395-402.

[33] Lenormand R, Zarcone C. Role of roughness and edges during imbibition in square capillaries. SPE Annual Technical Conference and Exhibition, 1984, SPE-13264.

[34] Dong M, Chatzis I. The imbibition and flow of a wetting liquid along the corners of a square capillary tube. Journal of Colloid and Interface Science, 1995, 172, 278-288.

[35] Delamarche E, Juncker D, Schmid H. Microfluidics for processing surfaces and miniaturizing biological assays. Advanced Materials, 2005, 17, 2911-2933.

[36] Safavieh R, Juncker D. Capillarics: Pre-programmed, self-powered microfluidic circuits built from capillary elements. Lab on a Chip, 2013, 13, 4180-4189.

[37] Chu R C, Simons R E, Ellsworth M J, Schmidt R R, Cozzolino V. Review of cooling technologies for computer products. IEEE Transactions on Device and Materials Reliability, 2004, 4, 568-585.

[38] Hancock M J, Sekeroglu K, Demirel M C. Bioinspired directional surfaces for adhesion, wetting and transport. Advanced Functional Materials, 2012, 22, 2223-2234.

[39] Heitz J, Plamadeala C, Wiesbauer M, Freudenthaler P, Wollhofen R, Jacak J, Klar T A, Magnus B, Köstner D, Weth A, Baumgartner W, Marksteiner R. Bone-forming cells with pronounced spread into the third dimension in polymer scaffolds fabricated by two-photon polymerization. Journal of Biomedical Materials Research Part A, 2016, 105A, 891-899.

[40] Coenjarts C A, Ober C K. Two-photon three-dimensional 
microfabrication of poly (dimethylsiloxane) elastomers. Chemistry of Materials, 2004, 16, 5556-5558.

[41] Michel B, Bernard A, Bietsch A, Delamarche E, Geissler M, Juncker D, Kind H, Renault J P, Rothuizen H, Schmid H, Schmidt-Winkel P, Stutz R, Wolf H. Printing meets lithography: Soft approaches to high-resolution patterning. IBM Journal of Research \& Development, 2002, 56, 527-542.

[42] Mujahid A, Iqbal N, Afzal A. Bioimprinting strategies: From soft lithography to biomimetic sensors and beyond. Biotechnology Advances, 2013, 31, 1435-1447.

[43] Kim P, Kwon K W, Park M C, Lee S H, Kim S M. Soft lithography for microfluidics: A Review. Biochip Journal, 2008, 2, 1-11.

[44] Becker H, Heim U. Hot embossing as a method for the fabrication of polymer high aspect ratio structures. Sensors and Actuators, A: Physical, 2000, 83, 130-135.

[45] Taniguchi J, Yoshikawa H, Tazaki G, Zento T. High-density pattern transfer via roll-to-roll ultraviolet nanoimprint lithography using replica mold. Journal of Vacuum Science \& Technology B: Microelectronics and Nanometer Structures, 2012, 30, 06FB07.

[46] Li Y, Ng H W, Gates B D, Menon C. Material versatility using replica molding for large-scale fabrication of high aspect-ratio, high density arrays of nano-pillars. Nanotechnology, 2014, 25, 285303.

[47] Micheal I J, Vidyasagar A J, Bokara K K, Mekala N K, Asthana A, Rao C M. Foil assisted replica molding for fabrication of microfluidic devices and their application in vitro. Lab on a chip, 2014, 14, 3695-3699. 